\title{
Microsatellite analysis of environmental and clinical isolates of the opportunist fungal pathogen Aspergillus fumigatus
}

\author{
KIMBERLY ROSEHART, MIRIAM H. RICHARDS and MICHAEL J. BIDOCHKA
}

Department of Biological Sciences, Brock University, St Catharines, Ontario, Canada L2S 3A1

\begin{abstract}
Microsatellite analysis was used to examine the genetic relatedness of 111 clinical and environmental isolates of the opportunist human pathogenic fungus Aspergillus fumigatus from Ontario, Canada. Forty-three A. fumigatus isolates were from clinical sources and 68 from environmental sources. Phylogenetic analysis of the genotypes revealed that there were no geographical or temporal associations of clinical or environmental genotypes. In fact, several of the environmental and clinical isolates showed identical (clonal) genotypes from disparate geographical areas. However, a locus by locus examination revealed that there were several significant differences in allele frequencies between clinical and environmental isolates. There may be linkage of certain microsatellite loci with genes affecting virulence in $A$. fumigatus. A susceptible individual may be equally predisposed to infection by any isolate of $A$. fumigatus. However, under transient selection as a pathogen, genes encoding alleles for enhanced virulence may not assort independently from microsatellite loci. A dynamic equilibrium may exist between random recombination of loci in the natural environment and selection for virulence factors during host infection cycles.
\end{abstract}

\section{Introduction}

It is estimated that each person inhales 5-5000 conidia of the opportunist fungal pathogen Aspergillus fumigatus daily [1]. This fungus produces and releases many $\left(10^{4}\right.$ conidia per conidial head) very small conidia (2$3 \mu \mathrm{m}$ in diameter) [1]. Given the small size of conidia, once airborne they can remain buoyant for extended periods. Indoors, such as in a hospital, conidia may persist for $>6$ months [2].

Once inhaled, conidia are eliminated by most individuals; however, immunocompromised individuals are at greatest risk. Conidia can colonise the upper respiratory tract, causing pulmonary infections including bronchopulmonary aspergillosis, aspergilloma and invasive aspergillosis [3-6]. From 1992 to 1994 the Canadian Infectious Disease Society enumerated nosocomial fungal infections from centres across the country, documenting 787 cases related to A. fumigatus

Received 11 March 2002; revised version accepted 6 Aug. 2002.

Corresponding author: Dr M. J. Bidochka (e-mail: bidochka @ spartan.ac.brocku.ca). of which $65 \%$ were fatal, and mortality was attributed to fungal infection in $85 \%$ of cases [7].

Outbreaks of nosocomial invasive aspergillosis, and the high fatalities associated with infection, have led to an effort to identify factors involved in the virulence of $A$. fumigatus and the identification and characterisation of highly virulent isolates. Are certain clinical isolates more virulent and genetically distinct from other isolates, or is infection by A. fumigatus simply a matter of contracting infection from any environmental source? Leenders et al. [6] characterised five clinical isolates obtained over a 2-month period and found that they were genetically distinct from each other and from environmental isolates. Tang et al. [8] found that clinical isolates were distinct from environmental isolates; however, only 2 clinical and 11 environmental isolates were characterised. Mondon et al. [9, 10] identified a $0.95-\mathrm{kb}$ PCR-amplified DNA fragment in A. fumigatus isolates associated with virulence in a murine model. On the other hand, Bart-Delabesse et al. [11] found no genetic differences between clinical and environmental isolates. Similarly, Debeaupuis et al. [12] have shown that no particular genotype was associated with human disease. 
Most of the studies that have been done on the population genetics of $A$. fumigatus have investigated European samples $[2,9-11,13,14]$. The present study examined genetic differences between environmental isolates of $A$. fumigatus collected in Ontario, Canada and clinical isolates from the same region. To examine the population genetic structure of A. fumigatus, microsatellite analysis was used with primers developed by Bart-Delabesse et al. [11].

\section{Materials and methods}

\section{Fungal isolates}

Isolates of A. fumigatus were from two sources, environmental and clinical. Environmental isolates were obtained near Peterborough, Ontario, Canada by sampling air. Air samples were collected weekly from 17 March to 27 Oct. 1999 with a Biotest RCS (Hycon) air sampler where conidia were impacted on to Rose Bengal agar strips. Sampling times ranged from $8 \mathrm{~min}$ to 2 min depending on the number of spores collected the week before; the more spores collected, the shorter the sampling time. The agar strip was incubated at $45^{\circ} \mathrm{C}$ to isolate A. fumigatus. These were identified microscopically and 68 isolates from the environmental sources were used for microsatellite analysis. Fortythree clinical isolates were kindly provided by $\mathrm{Dr}$ R. Summerbell (Chief Medical Mycologist in Ontario). These were isolated from infected patients in hospitals in the Ontario region and Table 1 outlines the region of Ontario from which each isolate was obtained.

\section{DNA isolation and microsatellite analysis}

Conidia of $A$. fumigatus were inoculated into flasks containing $50 \mathrm{ml}$ of sucrose broth (sucrose $2 \%$, peptone $1 \%$, yeast extract $0.3 \%, \mathrm{NaCl} 0.5 \%$ at $\mathrm{pH} 6.8-7.0$ ). Each flask was incubated overnight at $45^{\circ} \mathrm{C}$ in an incubator shaker set at $200 \mathrm{rpm}$. Mycelia were collected under vacuum filtration on to glass fibre filters and stored at $-80^{\circ} \mathrm{C}$. Frozen mycelia were ground to a fine powder with liquid nitrogen and a mortar and pestle.

DNA was extracted with the QIAGEN mini blood and tissue extraction kit, following the protocol outlined for tissue extraction. To obtain a sufficient quantity of DNA, the amount of ground mycelium used in the extraction procedure was doubled and the QIAGEN protocol was adjusted accordingly. DNA concentration and integrity were checked by electrophoresis with agarose $0.8 \%$ with TBE buffer.

Genetic variability between the environmental and clinical isolates of A. fumigatus was determined by microsatellite analysis. Primers and amplification conditions were those used by Bart-Delabesse et al. [11]. PCR products were separated by electrophoresis on agarose $2.5-3.0 \%$ gels. Gels were photographed with a
Table 1. Geographic origin and year of collection for each of the clinical isolates of A. fumigatus assessed and cardinal direction within Ontario, Canada

\begin{tabular}{|c|c|c|}
\hline Isolate no. & Location & Cardinal direction \\
\hline FR1723-97 & Timmins & $\mathrm{N}$ \\
\hline FR1724-97 & Timmins & $\mathrm{N}$ \\
\hline FR1847-97 & Hamilton & $\mathrm{S}$ \\
\hline FR1862-97 & Thunder Bay & $\mathrm{N}$ \\
\hline FR1864-97 & Thunder Bay & $\mathrm{N}$ \\
\hline FR2298-97 & Scarborough & $\mathrm{S}$ \\
\hline FR2314-97 & Brampton & $\mathrm{S}$ \\
\hline FR2379-97 & Scarborough & $\mathrm{S}$ \\
\hline FR2406-97 & Sudbury & $\mathrm{N}$ \\
\hline FR2453-97 & Weston & $\mathrm{S}$ \\
\hline FR2743-97 & Windsor & $\mathrm{S} / \mathrm{W}$ \\
\hline FR2836-97 & Brampton & $\mathrm{S}$ \\
\hline FR2837-97 & Brampton & $\mathrm{S}$ \\
\hline FR2851-97 & Brampton & $\mathrm{S}$ \\
\hline FR2923-97 & Sudbury & $\mathrm{N}$ \\
\hline FR2924-97 & Sudbury & $\mathrm{N}$ \\
\hline FR2925-97 & Scarborough & $\mathrm{S}$ \\
\hline FR2963-97 & Willowdale & $\mathrm{S}$ \\
\hline FR2964-97 & Weston & $\mathrm{S}$ \\
\hline FR3016-97 & Orangeville & $\mathrm{S}$ \\
\hline FR3019-97 & Mississauga & $\mathrm{S}$ \\
\hline FR3021-97 & Brampton & $\mathrm{S}$ \\
\hline FR3044-97 & Sudbury & $\mathrm{N}$ \\
\hline FR3045-97 & Sudbury & $\mathrm{N}$ \\
\hline FR3024-97 & Toronto & $\mathrm{S}$ \\
\hline FR78-98 & Ottawa & $\mathrm{E}$ \\
\hline FR81-98 & Scarborough & $\mathrm{S}$ \\
\hline FR112-98 & Sudbury & $\mathrm{N}$ \\
\hline SF5519-97 & St Thomas & $\mathrm{S} / \mathrm{W}$ \\
\hline SF5964-97 & North York & $\mathrm{S}$ \\
\hline SF5978-97 & North York & $\mathrm{S}$ \\
\hline SF6053-97 & Sturgeon Falls & $\mathrm{N}$ \\
\hline SF6055-97 & Don Mills & $\mathrm{S}$ \\
\hline SF6078-97 & Willowdale & $\mathrm{S}$ \\
\hline SF6362-97 & Weston & $\mathrm{S}$ \\
\hline SF6702-97 & Newmarket & $\mathrm{S}$ \\
\hline SF6703-97 & Newmarket & $\mathrm{S}$ \\
\hline SF6737-97 & Weston & $\mathrm{S}$ \\
\hline SF7145-97 & Mississauga & $\mathrm{S}$ \\
\hline SF7903-97 & Toronto & $\mathrm{S}$ \\
\hline SF9056-97 & Willowdale & $\mathrm{S}$ \\
\hline SF102-98 & Toronto & $\mathrm{S}$ \\
\hline SF301-98 & Etobicoke & $\mathrm{S}$ \\
\hline
\end{tabular}

gel documentation system (Gel Doc 1000, BioRad) and the sizes of the PCR products were calculated by comparison with internal mol. wt standards.

\section{Statistical analysis}

With NTSYS, a similarity matrix was calculated based on the method of simple matching coefficient. The matrix was analysed for hierarchical clustering by the unweighted pair group method with arithmetic averages (UPGMA). Differences in allele banding pattern frequency for each primer set for clinical and environmental isolates were determined by $\chi^{2}$ analysis.

Relationships among the 82 unique genotypes were also examined by constructing an unrooted phylogenetic network based on genetic distance, by the neighbour-joining method as implemented in PAUP ver. 4.b10, treating the different alleles at each microsatellite locus as unordered traits [15]. Three 
characteristics were then mapped on to the network, i.e., whether the samples were clinical or environmental, their geographic location and year of collection. Phylogenetic signal for any of these three characteristics would be indicated by separation of the characteristics on to different branches of the network.

\section{Results}

\section{Microsatellite analysis}

Samples were assessed at four loci, $A, B, C$ and $D$, all of which were polymorphic. In these samples locus $A$ had four different alleles, locus $B$ had six different alleles, locus $C$ had four different alleles and locus $D$ had nine different alleles, as illustrated in Fig. 1.

\section{Statistical analysis}

Pairwise comparisons between all environmental and clinical isolates by microsatellite banding patterns were generated in NTSYS to create a similarity matrix. A dendrogram was produced based on the hierarchical UPGMA cluster analysis to examine how 111 isolates used for analysis were related to one another (Fig. 2). Some of the clinical and environmental isolates were identical at the four loci assessed and the probability of sharing all alleles was calculated from allele frequency data. Environmental isolate AFTU9 was identical to clinical isolates FR2963-97 and FR2406-97 (probability of $1.1 \times 10^{-4}$ ); environmental isolate AFTU10 was identical to clinical isolate FR1862-97 (probability of $6.5 \times 10^{-5}$ ); clinical isolate SF6053-97 was identical to environmental isolate AF0331FT (probability of $1.3 \times$ $10^{-4}$ ); clinical isolate FR3019-97 was identical to environmental isolate AFTU5 (probability of $2.2 \times$ $10^{-3}$ ); environmental isolate AFEC6 was identical to FR2837-97 (probability of $2.4 \times 10^{-4}$ ); clinical isolates FR1724-97 and F6055-97 were identical to environmental isolate AF0505EC15 (probability of $6.3 \times$ $10^{-3}$ ); and clinical isolate FR112-98 was identical to environmental isolate AF0715EC6 (probability of $\left.5.4 \times 10^{-5}\right)$.

A phylogenetic approach was used to investigate whether there was an association of microsatellite genotypes with environmental and clinical isolates and whether there was a phylogeographic or temporal association. There was no indication of any phyloge-
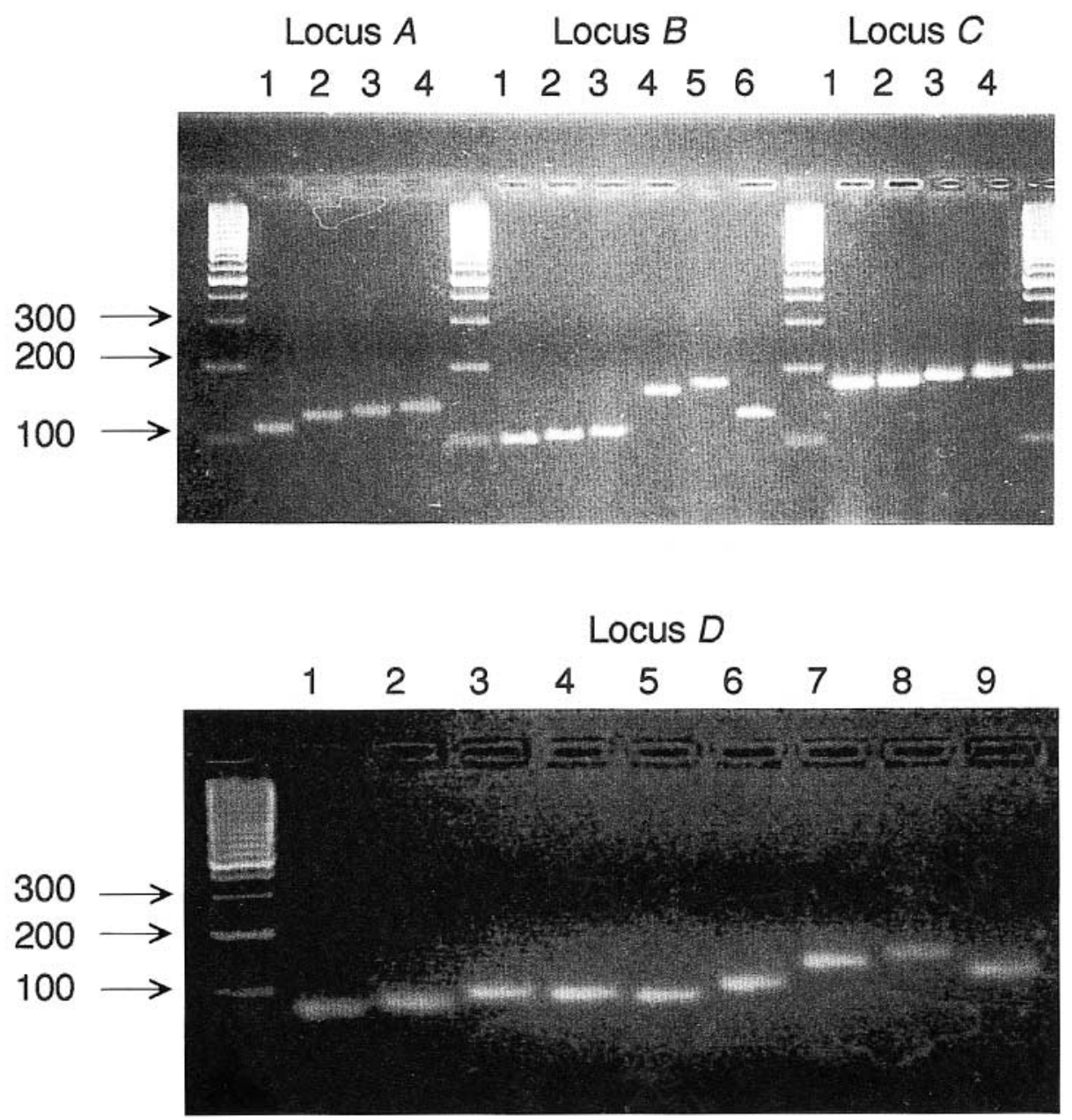

Fig. 1. Microsatellite-PCR banding patterns observed for 111 clinical and environmental isolates of $A$. fumigatus, amplified with primer A (4 alleles), primer B ( 6 alleles), primer C (4 alleles) and primer D ( 9 alleles). To the left of each primer set are the DNA markers (in bases). 


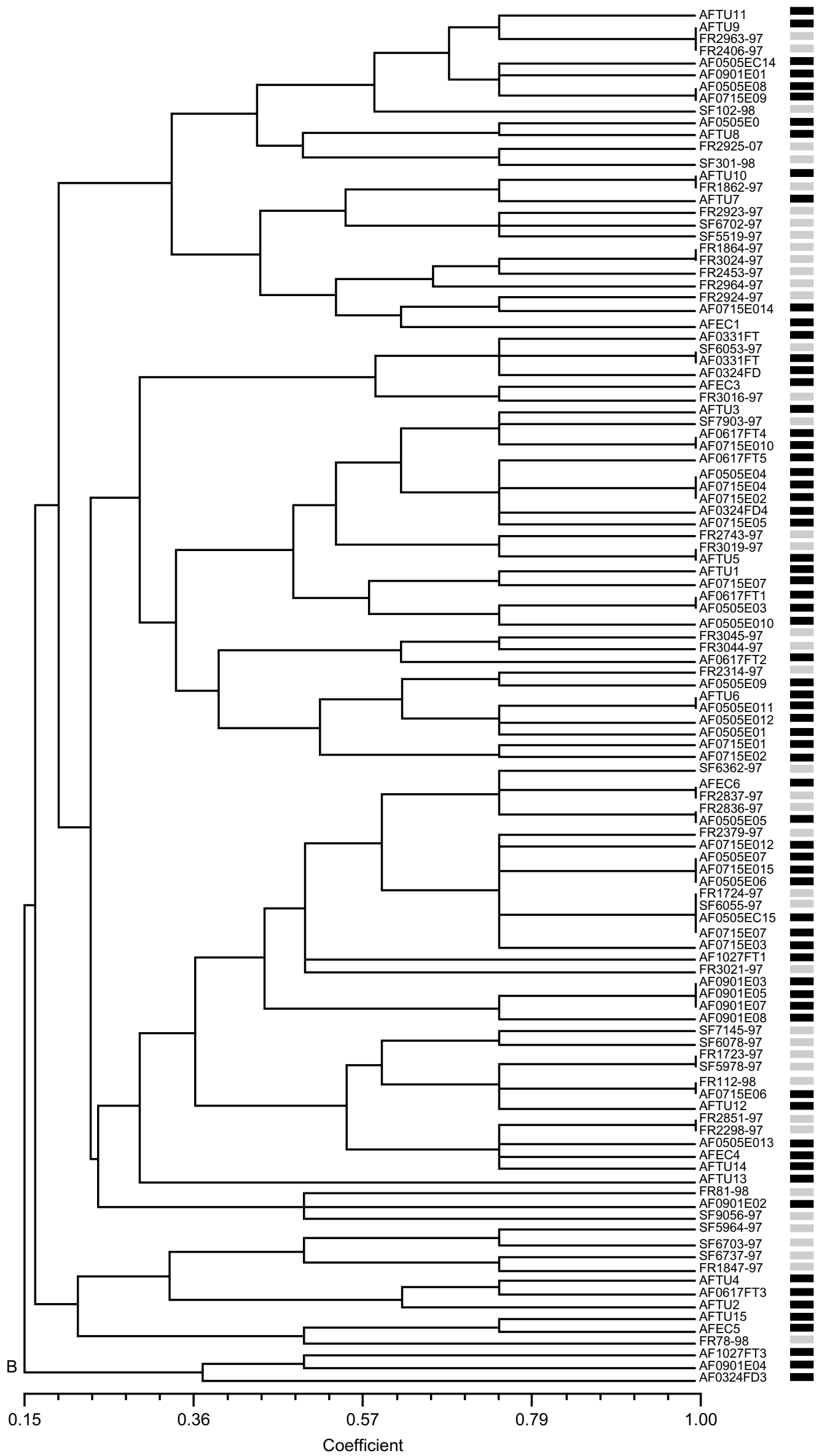

Fig. 2. Dendrogram illustrating the genetic relationships among the 43 clinical isolates and 68 environmental isolates of $A$. fumigatus. In the column to the right of the dendrogram a dark band indicates environmental isolates and a light band indicates clinical isolates. 
netic signal in clinical versus environmental samples (data not shown), as indicated by the fact that clinical and environmental groups were randomly dispersed across the entire network. Moreover, $7(8.5 \%)$ of 82 genotypes were found in both clinical and environmental samples. There was no association of genotypes with geography or year of collection. Lack of support for the separation of clinical and environmental genotypes was also suggested by parsimony and bootstrap analyses that produced $>312000$ shortest trees (length 8) with a completely unresolved consensus tree.

Fig. 3 shows the frequency distribution of alleles at each locus for clinical and environmental isolates. $\chi^{2}$ analyses were performed to examine whether alleles present at particular loci were significantly more prevalent in clinical or environmental isolates. There were significant differences in the alleles at locus $A$ when comparing clinical and environmental isolates $\left(\chi^{2}{ }_{(3)}=10.2618, \mathrm{p}=0.016\right)$. There were more clinical isolates with alleles $A 3$ and $A 4$ than in the environmental isolates and there were more environmental isolates with allele $A 2$. There were also differences in the distribution of alleles at locus $D$ between clinical and environmental isolates $\left(\chi^{2}{ }_{(8)}=24.1595, \quad \mathrm{p}=\right.$ $0.0022)$. There were more environmental isolates with alleles $D 4$ and $D 9$. Significant differences were not found for locus $B\left(\chi^{2}{ }_{(5)}=10.6914, \mathrm{p}=0.0578\right)$ or for locus $C\left(\chi_{(3)}^{2}=2.8114, \mathrm{p}=0.4216\right)$.

\section{Discussion}

Studies on the population genetics of A. fumigatus, when taken together, are inconclusive as to whether there are genotypic differences between clinical and environmental isolates $[2,6,8-11,13,14,16]$. Analyses in the present study showed that there were no broad genotypic differences between environmental and clinical isolates. The dendrogram of genetic relatedness showed that some clinical and environmental isolates of A. fumigatus were identical at the four microsatellite loci analysed.

Genetic similarity of the clinical and environmental isolates found in the present study, and those of previous studies, could be interpreted several ways. $A$.

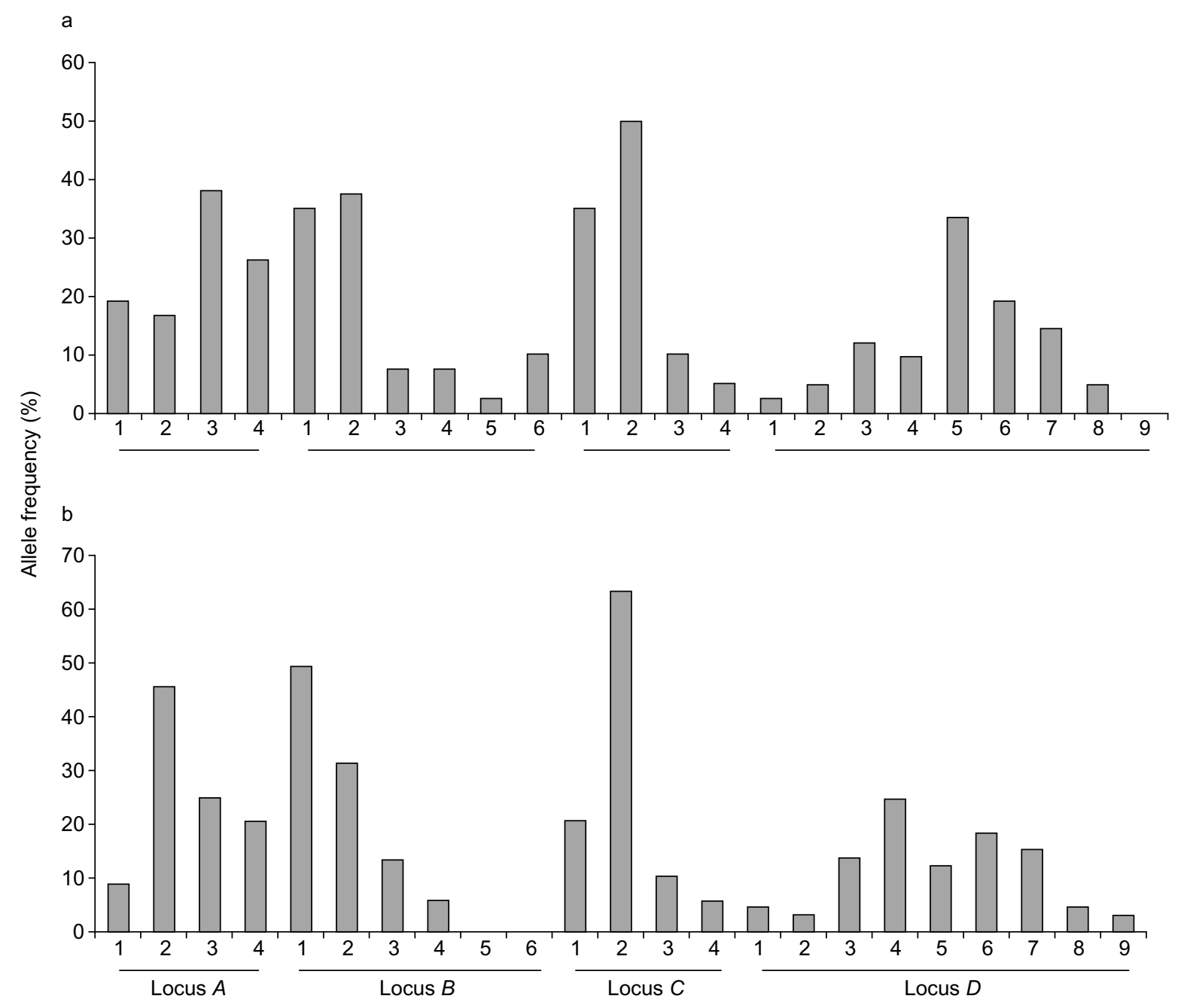

Fig. 3. Frequency of allele banding patterns for 111 isolates of $A$. fumigatus at each of the four loci $(A, B, C, D)$. The proportion of alleles showing different banding patterns for (a) the 43 clinical isolates, (b) the 68 environmental isolates. 
fumigatus is a saprophytic fungus that has properties such as thermotolerance and the ability to excrete hydrolytic extracellular enzymes that consequently allow opportunist colonisation of lung tissue [17]. Isolates that have additional properties that confer greater virulence may be represented in the sample of environmental isolates. Another explanation is that there is a stochastic component to infection. The present study did not have access to information pertaining to the identity or the health status of infected individuals from which the clinical $A$. fumigatus samples were isolated. A susceptible, immunocompromised individual may be equally predisposed to infection by any isolate of A. fumigatus.

Clinical isolates were not unique to a geographical region within Ontario nor were environmental isolates phylogeographically related. For example, several genotypically related clinical isolates were obtained from Thunder Bay, Sudbury, Newmarket, St Thomas, Weston and Toronto. The distance from Thunder Bay to Toronto is c. $1000 \mathrm{~km}$. Continuous exchange of conidia through air currents could explain the isolation of related genotypes from distant regions. However, geographical differences in the population genetics of A. fumigatus are not without precedent. For example, Katz et al. [18] identified an isolate of $A$. fumigatus that was unique to Australia.

Although there were no overall genotypic associations of clinical and environmental isolates, several microsatellite alleles were more predominant in either clinical or environmental isolates. These differences were not apparent in the phylogenetic analysis. The association of alleles with clinical isolates of $A$. fumigatus may indicate physical linkage to gene(s) involved in virulence. Mondon et al. $[9,10]$ identified a 0.95-kb PCR-amplified DNA fragment that was associated with virulence in a murine model. Although the fragment has not yet been identified as a virulence factor, it is possible that it may be linked to another gene that codes for a virulence factor. Katz et al. [18] found that there were differences in virulence among isolates of $A$. fumigatus. An ostrich infected with the isolate NSW3 responded positively to drug treatment while ostriches infected with a different isolate did not. Tsai et al. [19] identified a gene required for conidial pigmentation (alb1) which, when mutagenically disrupted, resulted in an albino conidial phenotype and showed a loss of virulence in a murine model.

Linkage between genes associated with virulence and microsatellite loci may be perpetuated in isolates of $A$. fumigatus because it is an asexual, haploid fungus. Even without selection for virulence the time required for linkage equilibrium to develop among loci would be much greater in Aspergillus than in a sexual organism. New combinations of unlinked loci would be generated by parasexual mechanisms: mutation plus vegetative hyphal fusion between genetically dissimilar types, karyogomy, mitotic recombination and spontaneous haploidisation. Given the rarity of these events, linked loci would tend to be maintained in a specific configuration. A dynamic equilibrium may exist between random recombination of loci in the natural environment and selection for virulence factors during host infection cycles.

The potential genetic differences between clinical and environmental isolates of $A$. fumigatus with respect to linked virulence and microsatellite loci illustrate the need for more research in this area. Differences in potential virulence factors between clinical and environmental isolates of $A$. fumigatus are currently being explored.

This research was supported by an operating grant from the National Sciences and Engineering Research Council of Canada (NSERC) to M.J.B. We thank Dr Alan Castle and an anonymous reviewer for their very helpful comments.

\section{References}

1. Latge JP, Debeaupuis JP, Sarfati J, Paris S. Variability in the human opportunistic fungal pathogen Aspergillus fumigatus. In: Bridge P, Couteaudier Y, Clarkson J (eds) Molecular variability of fungal pathogens. Wallingford, Oxon, CAB International. 1998.

2. Girardin H, Sarfati J, Traore E, Camet JD, Derouin F, Latge JP. Molecular epidemiology of nocosomial invasive aspergillosis. $J$ Clin Microbiol 1994; 32: 684-690.

3. Arruda LK, Mann BJ, Chapman MD. Selective expression of a major allergen and cytotoxin, Asp $f$ I, in Aspergillus fumigatus. Implications for the immunopathogenesis of Aspergillus related diseases. J Immunol 1992; 149: 2254-3359.

4. Castelanno DM, Green JN, Robinson LA, Sandin RL, MuroCacho CA. Pulmonary pseudoaneurysm due to Aspergillus fumigatus in a breast cancer patient. Inf Med 1999; 16: 201-204.

5. Kwong-Chung KJ, Bennett JE. Medical mycology. Philadelphia, Lea \& Febiger. 1992.

6. Leenders A, van Belkum A, Janssen S et al. Molecular epidemiology of apparent outbreak of invasive aspergillosis in a hematology ward. J Clin Microbiol 1996; 34: 345-351.

7. Nicolle LE, Rotstein C, Bourgault AM et al. Invasive fungal infections in Canada from 1992 to 1994. Can J Infect Dis 1998; 9: 347-352.

8. Tang CM, Cohen J, Rees AJ, Holden DW. Molecular epidemiological study of invasive pulmonary aspergillosis in a renal transplantation unit. Eur $J$ Clin Microbiol Infect Dis 1994; 13: 318-321.

9. Mondon P, De Champs C, Donadille A, Ambroise-Thomas P, Grillot R. Variation in virulence of Aspergillus fumigatus in a murine model of invasive pulmonary aspergillosis. $\mathrm{J} \mathrm{Med}$ Microbiol 1996; 45: 186-191.

10. Mondon P, Thelu J, Lebeau B, Ambroise-Thomas P, Grillot R. Virulence of Aspergillus fumigatus strains investigated by random amplified polymorphic DNA analysis. $J$ Med Microbiol 1995; 42: 299-303.

11. Bart-Delabesse E, Humbert JF, Delabesse E, Bretagne S. Microsatellite markers for typing Aspergillus fumigatus isolates. J Clin Microbiol 1998; 36: 2413-2418.

12. Debeaupuis J-P, Sarfati J, Chazalet V, Latge J-P. Genetic diversity among clinical and environmental isolates of Aspergillus fumigatus. Infect Immun 1997; 65: 3080-3085.

13. Aufauvre-Brown A, Cohen J, Holden DW. Use of randomly amplified polymorphic DNA markers to distinguish isolates of Aspergillus fumigatus. J Clin Microbiol 1992; 30: 2991-2993.

14. Rodriguez E, De Meeus $\mathrm{T}$, Mallie M et al. Multicentric epidemiological study of Aspergillus fumigatus isolates by multilocus enzyme electrophoresis. J Clin Microbiol 1996; 34: 2559-2568. 
15. Swofford DL. PAUP ver 4.0b10, copyright 2001 Sinauer Associates.

16. Girardin H, Latge JP, Srikantha T, Morrow B, Soll DR Development of DNA probes to fingerprinting Aspergillus fumigatus. J Clin Microbiol 1993; 31: 1547-1554.

17. St Leger RJ, Screen SE. In vitro utilization of mucin, lung polymers, plant cell walls and insect cuticle by Aspergillus fumigatus, Metarhizium anisopliae and Haematoncetria haem- atococca. Mycol Res 2000; 104: 463-471.

18. Katz ME, Mcloon M, Burrows S, Cheetham BF. Extreme DNA sequence variation in isolates of Aspergillus fumigatus. FEMS Immunol Med Microbiol 1998; 20: 283-288.

19. Tsai HF, Chang YC, Washburn RG, Wheeler MH, KwonChung KJ. The developmentally regulated alb1 gene of Aspergillus fumigatus: its role in modulation of conidial morphology and virulence $J$ Bacteriol 1998; 180: 3031-3038. 